\title{
Conduite à tenir face à une plaie perforante du plancher buccal
}

\section{Action to be taken against a puncture wound of oral floor}

\section{Résumé}

\section{MOTS-CLEFS :}

- Plancher buccal, plaie perforante, conduite ò tenir

\section{KEYWORDS:}

- Oral floor, puncture wound, action to be taken against

AOS 2014;269:42-46 DOI: $10.1051 / \mathrm{aos} / 2014308$ C EDP Sciences 2014
Après un bref rappel sur les structures anatomiques composant le plancher buccal, nous verrons quelles sont les complications possibles en fonction des structures endommagées.

Quelle est la conduite à adopter en urgence? Et comment réparer au mieux les lésions ?

A partir du «bilan lésionnel initial » nous proposerons une conduite à tenir spécifique permettant d'améliorer le pronostic.

\section{Abstract}

After a brief recall focused on the anatomical structures of the oral floor we will see which complications can occur according to the damaged structures.

Which action has to be taken in emergency? And how to manage the wound at best?

According to the "initial assessment of the lesions » we will propose a course of action allowing to improve the prognosis.

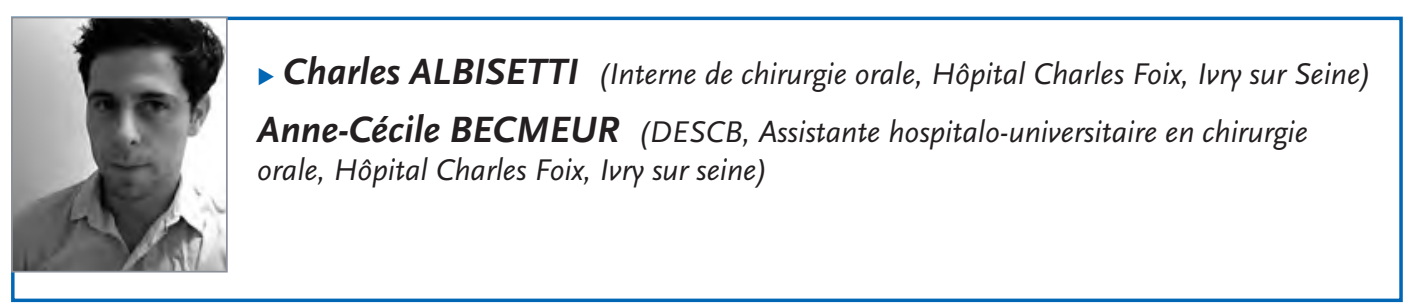




\section{INTRODUCTION}

La survenue d'une plaie du plancher buccal est une complication relativement fréquente lors de la pratique d'un acte de chirurgie orale ou encore lors d'une préparation périphérique de la face linguale d'une molaire mandibulaire. En effet, il peut arriver qu'un instrument dérape, provoquant alors une plaie plus ou moins perforante souvent très hémorragique et à proximité de structures nobles telles une artère, un nerf ou encore un canal excréteur d'une glande salivaire. Cette complication aïgue vient alors perturber le bon déroulement du soin en cours et peut être source de stress pour le praticien non familier avec l'anatomie de cette région et la conduite à tenir en urgence.

L'objectif de cet article est de familiariser le praticien à l'anatomie du plancher buccal pour l'aider à diagnostiquer les complications et donc pratiquer le «bilan lésionnel » et enfin de proposer une conduite à tenir en urgence et à distance face à une plaie perforante du plancher buccal.

\section{LA RÉGION \\ DU PLANCHER BUCCAL : RAPPELS ANATOMIQUES [4]}

\section{Les principaux muscles}

Le muscle mylo-hyoïdien pair et symétrique, c'est une fine nappe musculaire triangulaire à base postérieure qui s'insère en haut et en avant sur la face postérieure du corps de la mandibule, sur la ligne oblique interne ou mylo-hyoïdienne et en bas sur l'os hyoïde. Les deux muscles sont unis sur la ligne médiane par le raphé médian. Il est innervé par le nerf mylo-hyoïdien branche du nerf alvéolaire inférieur. Ce muscle est abaisseur de la mandibule.

Le muscle génio-hyoïdien est un muscle pair, symétrique, court et épais, situé sous la langue donc en position para-médiane. Il s'insère sur la face postérieure de la symphyse mandibulaire (sur l'apophyse géni inférieure). Il se dirige en bas et en arrière en s'élargissant progressivement pour se terminer sur la face antérieure du corps de l'os hyoïde. Il est innervé par le nerf génio-hyoïdien branche du grand hypoglosse. C'est un abaisseur de la mandibule.

Le muscle génioglosse pair et symétrique, c'est le plus volumineux muscle extrinsèque de la langue. Il s'insère en avant à la face interne de la symphyse mandibulaire et s'étend jusqu'à l'os hyoïde. Il est innervé par le nerf grand hypoglosse et vascularisé par l'artère linguale. Son action est de tirer la langue vers l'avant et vers le bas.

\section{La vascularisation du plancher buccal}

L'artère linguale est la $3^{\text {ème }}$ branche de l'artère carotide externe (entre l'artère thyroïdienne supérieure et l'artère faciale). Elle vascularise la langue et la glande sublinguale par l'intermédiaire de l'artère sublinguale. Son trajet chemine jusqu'à la pointe de la langue en passant entre les muscles génioglosse et mylo-hyoïdien.

L'artère sublinguale naît de l'artère linguale et chemine dans le plancher buccal vers l'avant pour aller vasculariser la glande sublinguale.

L'artère sous mentale est une collatérale de l'artère faciale. Elle naît au niveau de la glande sous maxillaire et se prolonge en avant suivant le bord inférieur de la mandibule. Elle vascularise la glande sous maxillaire, les muscles mylo-hyoïdien et digastrique et la région symphysaire mandibulaire.

Le retour veineux s'effectue depuis la langue par les veines ramiques, sublinguales et linguales.

\section{L'innervation du plancher buccal}

Le nerf lingual est un nerf sensitif branche du nerf mandibulaire V3. Son trajet, depuis la langue vers le nerf mandibulaire traverse la loge sublinguale entrant alors en rapport étroit avec le canal de Wharton qu'il sous croise en dessous et en dedans. Il sort alors du plancher buccal pour cheminer le long de la face linguale de la crête osseuse mandibulaire pour aller s'anastomoser au nerf alvéolaire inférieur. Son calibre varie de 3 à $7 \mathrm{~mm}$ et sa teinte est nacrée. Sa fonction est de transmettre les afférences sensitives et gustatives des $2 / 3$ antérieurs de la langue.

\section{Les glandes salivaires}

La glande submandibulaire est située dans la partie latérale et profonde du plancher buccal dans la loge submandibulaire et reposant sur le muscle mylo-hyoïdien. Elle entretient des rapports étroits avec le nerf lingual, le nerf hypoglosse, la branche inférieure du nerf facial et l'artère faciale. Son canal excréteur (canal de Wharton) traverse le plancher buccal pour créer un ostium latéralement au frein de la langue.

La glande sublinguale est située dans le plancher antérieur dans la loge sublinguale et entretient des rapports étroit avec le canal de Wharton et l'artère sublinguale. 


\section{QUELLES SONT LES \\ COMPLICATIONS POSSIBLES EN CAS DE PLAIE PERFORANTE DU PLANCHER BUCCAL? [1, 2]}

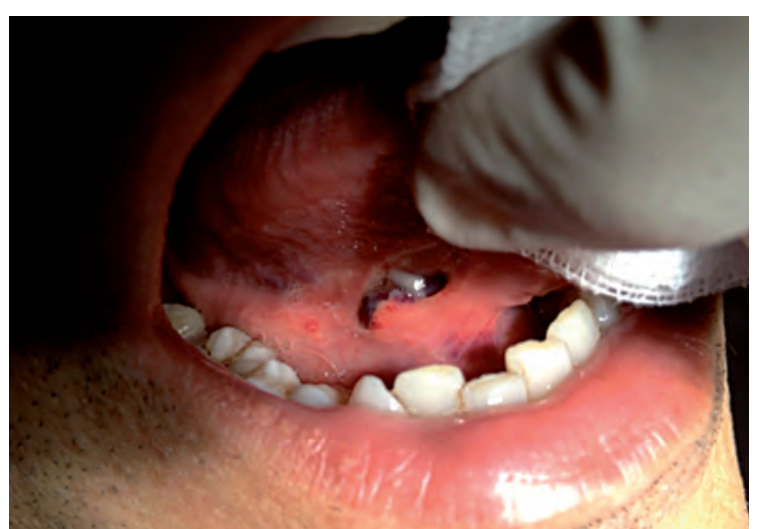

$\triangle$ Fig. 1.

Plaie opératoire pelvi-linguale après dérapage du syndesmotome faucille lors de la phase de luxation. Le nerf lingual est visible à travers la plaie. Ce patient n'a pas présenté d'hypoesthésie secondaire au traumatisme.

\begin{abstract}
A noter
Différents types de plaies existent :

$\checkmark$ les plaies par instruments chirurgicaux types élévateurs ou syndesmotomes. Il existe en général une plaie franche avec atteinte directe des structures localisées dans le champ de perforation.

$\checkmark$ les plaies par instruments rotatifs. Le mécanisme lésionnel sous-entend un arrachement, une dilacération et un étirement des tissus, qui peut rendre le bilan des lésions et la reconstruction par suture directe du site plus difficile. Elles sont de moins bon pronostic.
\end{abstract}

Lésion du nerf lingual lors d'une plaie postérieure Elle entraîne une perte des fonctions sensitive et sensorielle (perte du goût) des 2/3 antérieurs de la langue.

\section{Lésion d'une artère linguale, sublinguale ou sous mentale}

Elle entraîne une hémorragie aigue extériorisée. On la différencie d'une lésion veineuse par l'abondance du saignement caractéristique « en jet » pulsatile. Un hématome du plancher peut survenir secondairement.

\section{Lésion d'une veine linguale, sublinguale ou ramique}

Elle entraine une hémorragie aigue de plus faible abondance, en «nappe ». Un hématome du plancher peut survenir secondairement.

\section{Lésion d'une glande salivaire submandibulaire} ou sublinguale ou du canal excréteur de Wharton Vision directe du tissu glandulaire au travers de la perforation ou encore mise en évidence de la rupture du canal de Wharton avec un écoulement de salive «aberrant » au niveau de l'extrémité proximale. Une plaie directe du tissu glandulaire pourra entraîner une perte de fonction de la glande localisée ou généralisée, ou encore une rétention salivaire au niveau du tissu glandulaire lésé.

\section{Lésion musculaire du plancher buccal}

Elle entraîne en l'absence de suture des fibres musculaires un risque de déficit moteur selon le muscle considéré.

\section{Lésion superficielle de la muqueuse}

Sans retentissement direct après suture simple de la plaie.

\footnotetext{
A noter

La présence ou la suspicion d'un hématome du plancher buccal est une urgence vitale et doit conduire à un transfert immédiat du patient dans un service d'urgences médicales.

Ies signes cliniques:

I apparition d'une tuméfaction violacée du plancher buccal, I surélévation de la langue vers le palais,

I obstruction des voies aériennes supérieures,

I apparition d'une « dyspnée » inspiratoire,

I stress aigu pour le patient.

$\checkmark$ le traitement en urgence peut comprendre :

I une intubation naso-trachéale et ventilation mécanique en cas de signes de mauvaise tolérance respiratoire, cardiaque ou hémodynamique.

I une corticothérapie intra-veineuse à forte dose (but = diminuer l'œdème),

I une artério-embolisation de l'artère responsable pour stopper le saignement en service de radiologie interventionnelle (après identification de l'artère responsable grâce au scanner avec injection de produit de contraste iodé).
}

\section{COMMENT PRÉVENIR LA SURVENUE D'UN ACCIDENT TRAUMATIQUE DU PLANCHER BUCCAL ? [1, 2, 3]}

\section{Chirurgie pratiquée à 4 mains}

L'aide opératoire découvre le champ opératoire au chirurgien et protège la langue et le plancher buccal à l'aide d'un miroir en cas d'utilisation d'un instrument rotatif.

\section{Utilisation d'une compresse en contre-appui} Lors de l'utilisation d'un élévateur ou d'un syndesmotome lors de la phase de luxation. Le dérapage éventuel de l'instrument peut être contenu par le doigt protégé d'une compresse placé en antagoniste. 
L'apprentissage des points d'appui

La stabilité de la main de l'opérateur utilisant un instrument rotatif est capitale afin de contrôler un mouvement de dérapage.

\section{QUELLE EST LA CONDUITE IMMÉDIATE À TENIR FACE À UNE PLAIE PERFORANTE DU PLANCHER BUCCAL? [2, 3]}

Rester calme. Le caractère soudain du traumatisme, la douleur aigue ressentie par le patient dans une zone non anesthésiée, la sensation de « raté » par le chirurgien amènera une situation de crise que l'opérateur devra gérer avec «sang-froid» afin de réparer le traumatisme commis.

Rassurer le patient. Afin d'obtenir sa coopération au fauteuil pour la suite du traitement.

Demander de l'aide. Rechercher l'aide d'un praticien qualifié en chirurgie orale ou aide opératoire supplémentaire si nécessaire.

Comprimer et tamponner la zone à l'aide d'une compresse stérile. Permettant de contenir une éventuelle hémorragie aigue, favoriser l'hémostase et optimiser la visualisation de la plaie.

Pratiquer une anesthésie de la plaie. Avec vasoconstricteurs en l'absence de contre-indication. NB : système d'injection aspiratif pour vérifier l'absence d'injection dans un vaisseau.

Désinfecter le site à l'aide d'une solution antiseptique. Type chlorhexidine $0,12 \%$ ou bétadine buccale.

Puis, faire le bilan lésionnel de la plaie avant la reconstruction.

\section{En cas d'hémorragie aigue par plaie artérielle ou veineuse}

Maintenir la compression pendant environ 5 à 10 minutes afin d'obtenir une hémostase locale. Si besoin : acide tranexamique $\left(\right.$ Exacyl $\left.^{\circledR}\right) 1$ ampoule sur compresse stérile.

\Evaluer le risque hémorragique. Rechercher une anomalie de la coagulation innée ou acquise, une thrombopénie, un traitement antiagrégant plaquettaire ou anticoagulant (si AVK : obtenir le dernier INR).

I Si visualisation de l'artère ou veine responsable, liga- ture artérielle ou veineuse par un point en X autour du moignon proximal responsable du saignement à l'aide d'un fil résorbable type Vicryl 3.0 ou 4.0. ou électrocoagulation du moignon à l'aide d'une pince bipolaire.

Utilisation possible de matériaux hémostatiques résorbables type treillis d'oxycellulose (Surgicel $^{\circledR}$ ) pour combler le site avant la suture.

I Suture par points séparés de la plaie après vérification de l'absence de saignement.

ISi absence d'obtention de l'hémostase locale, contacter un service d'urgences médicales.

\section{En cas de suspicion de plaie du nerf lingual}

I Tester le nerf lingual. Rechercher l'apparition d'une hypoesthésie voir une anesthésie ou encore des paresthésies sur les $2 / 3$ antérieurs de la langue homolatérale à la lésion. $N B$ : il est impossible de tester la sensibilité du nerf lingual en présence d'une anesthésie tronculaire homolatérale.

I Si présence d'une section du nerf lingual avec anesthésie complète, adresser le patient en urgence dans un service de chirurgie maxillo-faciale pour suture micro-chirurgicale du nerf lingual sous microscope .

I Si présence d'une hypoesthésie avec suspicion de lésion partielle du nerf lingual :

I suture de la plaie

I corticothérapie post opératoire : Solupred $1 \mathrm{mg} / \mathrm{kg} / \mathrm{j}$ le matin pendant 5 jours

Ivitaminothérapie B1 et B6 : 2cp matin midi et soir pendant 1 mois et vitamine $B 12: 1$ ampoule le matin pendant 1 mois (NB: aucune étude scientifique n'a démontré l'efficacité de la vitaminothérapie sur les lésions nerveuses chez l'homme)

Ila prise en charge d'une éventuelle douleur neuropathique fera appel à 2 molécules : l'Amitriptyline (Laroxyl $l^{\circledast}: 25$ à 100mg par jour) ou la Gabapentine (Neurontin ${ }^{\circledast}$ )

Ila surveillance de la récupération nerveuse sera reportée précisément dans le dossier médical à l'aide d'une cartographie ou d'un shéma.

\section{En cas de plaie d'une glande salivaire ou de son canal excréteur}

Visualisation directe du tissu glandulaire lésé ou suspicion d'effraction de la loge submandibulaire ou sublinguale

I Fermeture de la loge glandulaire et isolement de la glande du milieu septique buccal par des points séparés au fil résorbable +/- antibioprophylaxie par amoxicilline $2 \mathrm{~g} / \mathrm{j} 7$ jours en l'absence de contre-indication. 
I Consultation de contrôle à 10 jours. Vérifier le bon fonctionnement de la glande par les tests cliniques correspondants, rechercher une rétention salivaire par palpation bidigitale de la glande à la recherche d'une tuméfaction douloureuse, masser la glande et rechercher un écoulement à l'ostium du Wharton et préciser sa nature.

I La glande submandibulaire entretenant des rapports étroits avec le nerf lingual, hypoglosse, la branche inférieure du nerf facial, l'artère faciale et l'artère linguale, il convient de rechercher une lésion associée.

I Des investigations complémentaires (ex : Sialendoscopie de la glande sub-mandibulaire) seront demandées en fonction du bilan lésionnel initial.

\section{Plaie du canal de Wharton}

ILa ré-anastomose du canal de Wharton sectionné ne pourra être tentée qu'en présence d'une plaie franche par objet tranchant avec visualisation correcte des deux fragments, avec utilisation d'aide optique et fil fin 6.0. NB : la sténose cicatricielle doit être prévenue par une plastie d'élargissement des 2 fragments anastomosés.

ISi la ré-anastomose est impossible, tenter d'aboucher la partie proximale du canal à la muqueuse afin de créer un «néo-ostium».

\section{CONCLUSION}

La connaissance de l'anatomie du plancher buccal et de la conduite à tenir face à une perforation accidentelle dans cette région permet au praticien de gérer cette complication opératoire dans le respect des structures nobles qui y résident.

\section{Bibliographie}

[1] Bouletreau P., Froget N., Gleizal A. et coll. Affections du plancher de la bouche. EMC, Médecine buccale, 28-285-V-10, 2008.

[2] Boucher Y., Cohen E. Urgences dentaires et médicales conduite à tenir : Prévention chez le patient à risque. 2007. $E d C d P$

[3] Robinson PP, LoescherAR, Yates J. et coll. Current managment of damage to the inferior alveolar and lingual nerves as a result of removal of third molars. Br J Oral Maxillo-fac Surg 2004 : 42 : 285-92

[4] Putz P, Pabst, Atlas d'anatomie humaine Sobotta tome 1 tête, cou, membre supérieur. 2000. Editions médicales internationales.

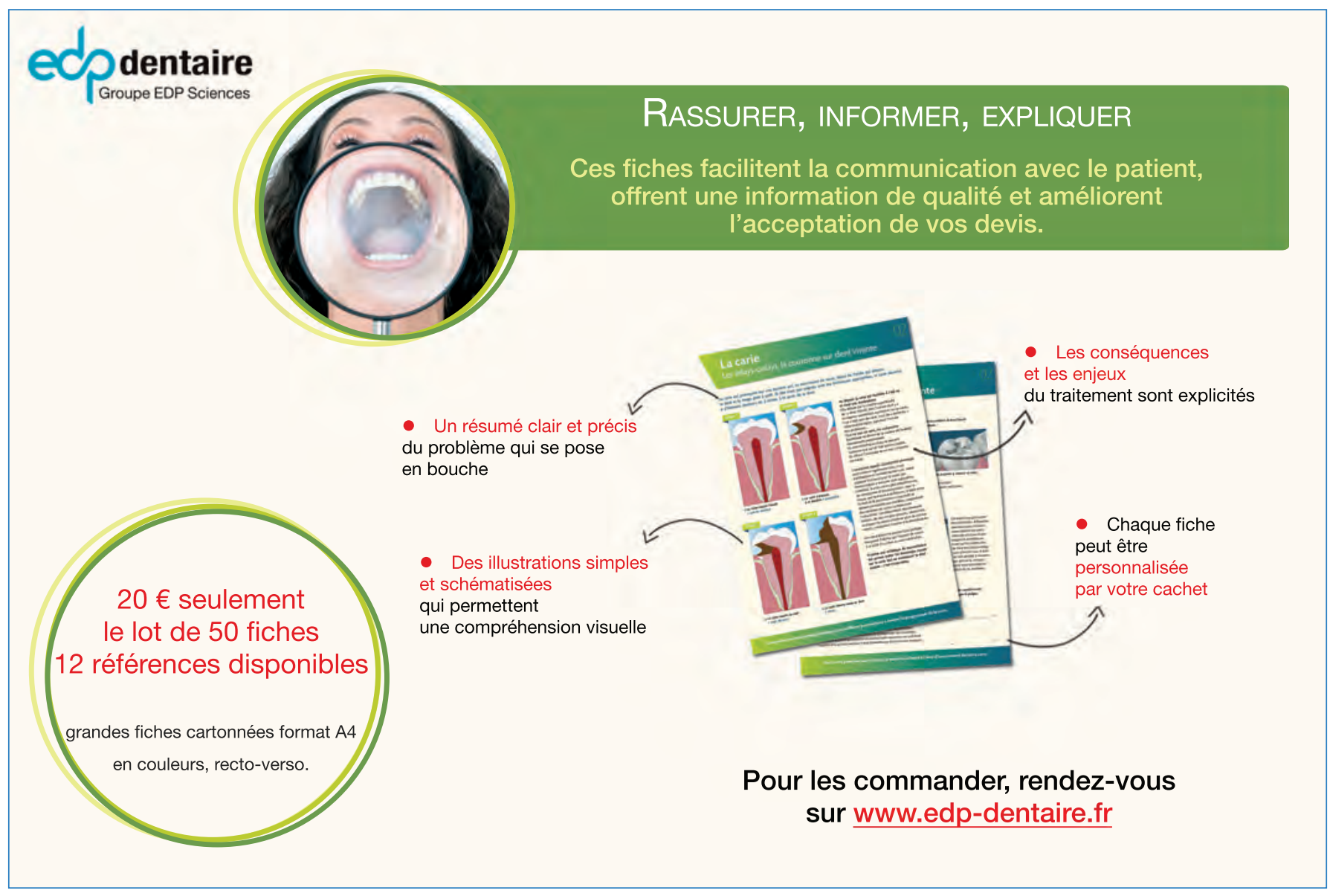

